\title{
Identification and validation of a six-gene signature associated with glycolysis to predict the prognosis of patients with cervical cancer
}

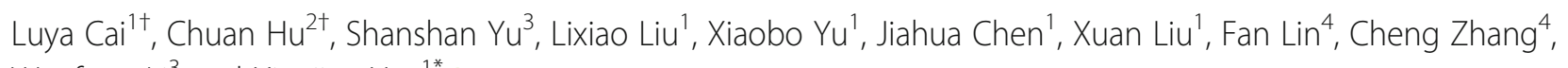
Wenfeng $\mathrm{Li}^{3}$ and Xiaojian $\operatorname{Yan}^{1 *}$ (i)

\begin{abstract}
Background: Cervical cancer (CC) is one of the most common gynaecological cancers. The gene signature is believed to be reliable for predicting cancer patient survival. However, there is no relevant study on the relationship between the glycolysis-related gene (GRG) signature and overall survival (OS) of patients with CC.

Methods: We extracted the mRNA expression profiles of 306 tumour and 13 normal tissues from the University of California Santa Cruz (UCSC) Database. Then, we screened out differentially expressed glycolysis-related genes (DEGRGs) among these mRNAs. All patients were randomly divided into training cohort and validation cohort according to the ratio of 7: 3. Next, univariate and multivariate Cox regression analyses were carried out to select the GRG with predictive ability for the prognosis of the training cohort. Additionally, risk score model was constructed and validated it in the validation cohort.

Results: Six mRNAs were obtained that were associated with patient survival. The filtered mRNAs were classified into the protective type (GOT1) and the risk type (HSPA5, ANGPTL4, PFKM, IER3 and PFKFB4). Additionally, by constructing the prognostic risk score model, we found that the OS of the high-risk group was notably poorer, which showed good predictive ability both in training cohort and validation cohort. And the six-gene signature is a prognostic indicator independent of clinicopathological features. Through the verification of PCR, the results showed that compared with the normal cervial tissuses, the expression level of six mRNAs were significantly higher in the CC tissue, which was consistent with our findings.

Conclusions: We constructed a glycolysis-related six-gene signature to predict the prognosis of patients with CC using bioinformatics methods. We provide a thorough comprehension of the effect of glycolysis in patients with CC and provide new targets and ideas for individualized treatment.
\end{abstract}

Keywords: Cervical cancer, Glycolysis, Prognosis, Gene signature

\footnotetext{
* Correspondence: yxjbetter2016@hotmail.com

${ }^{\dagger}$ Luya Cai and Chuan Hu contributed equally to this work.

'Department of Obstetrics and Gynecology, The First Affiliated Hospital of

Wenzhou Medical University, 2 Fuxue Road, Wenzhou, Zhejiang 325000, P.R.

China

Full list of author information is available at the end of the article
}

(c) The Author(s). 2020 Open Access This article is licensed under a Creative Commons Attribution 4.0 International License, which permits use, sharing, adaptation, distribution and reproduction in any medium or format, as long as you give appropriate credit to the original author(s) and the source, provide a link to the Creative Commons licence, and indicate if changes were made. The images or other third party material in this article are included in the article's Creative Commons licence, unless indicated otherwise in a credit line to the material. If material is not included in the article's Creative Commons licence and your intended use is not permitted by statutory regulation or exceeds the permitted use, you will need to obtain permission directly from the copyright holder. To view a copy of this licence, visit http://creativecommons.org/licenses/by/4.0/ The Creative Commons Public Domain Dedication waiver (http://creativecommons.org/publicdomain/zero/1.0/) applies to the data made available in this article, unless otherwise stated in a credit line to the data. 


\section{Background}

Cervical cancer (CC) is one of the most common gynaecological cancers, accounting for the fourth cause of cancer-related death in women [1]. According to global cancer statistics, in 2018, there were nearly 570 thousand new cases of CC worldwide, with approximately 310 thousand deaths [1]. In recent years, with the launch of the human papillomavirus (HPV) vaccination programme, the incidence of $\mathrm{CC}$ in developed countries has significantly decreased, but in developing countries, it is still on the rise [2], and the age of onset tends to be younger. In addition, a large proportion of patients with CC are found to be in an advanced stage, and at this time, treatment options are extremely limited, and side effects are more serious, with a 5-year survival rate of less than $20 \%$ [3-5]. Moreover, patients with the same clinical stage and pathological type tend to adopt the same treatment, but the prognosis of patients is different, which is mainly due to the genetic heterogeneity of patients. Therefore, it is necessary to identify effective biomarkers to predict the prognosis of patients with $\mathrm{CC}$.

Aerobic glycolysis is a special mechanism of tumour cell metabolism, also known as the Warburg effect [6], which plays an important role in promoting the growth and metastasis of various tumours, including CC. Some studies have found that HPV protein can promote the development of cancer through the Warburg effect, and the Warburg effect may also contribute to the enhancement of virus replication ability in the early stage of HPV infection [7]. In addition, total lesion glycolysis (TLG) is a measure of tumour metabolic activity, and some retrospective studies have found that TLG was significantly related to the recurrence-free survival (RFS) and overall survival (OS) of patients with CC [8-10]. Moreover, some glycolytic enzymes have also been proven to be related to the prognosis of CC. For example, hexokinase-2 (HK2), an enzyme that catalyses the conversion of glucose into glucose-6-phosphate, is overexpressed in a variety of cancers, has a promoting effect on the occurrence and development of CC, and is significantly related to the prognosis of patients [11]. Lactate dehydrogenase A (LDHA) and phosphofructokinase $\mathrm{P}$ (PFKP) were found to be significantly correlated with progression-free survival (PFS) and OS in patients with $\mathrm{CC}$, and the expression level of LDHA in recurrent tumours was significantly higher than that in nonrecurrent tumours [12]. Glyceraldehyde-3-phosphate dehydrogenase $(\mathrm{GAPDH})$ is a classical glycolytic enzyme that has been reported to be significantly increased in CC [13]. However, single gene biomarkers cannot achieve a good prediction effect, and some studies have suggested that gene signatures may be a better choice for predicting patient outcomes.
By mining public databases, many relevant studies have found that the glycolysis-related gene (GRG) signature is closely associated with the prognosis of patients with cancer, such as lung adenocarcinoma [14], liver cancer [15], pancreatic ductal carcinoma [16] and endometrial carcinoma [17]. Nevertheless, there is no bioinformatics research on this in CC. Thus, in this study, we analysed the relationship between the GRG signature and $\mathrm{CC}$ through the University of California Santa Cruz (UCSC) Database, which helped us better assess the prognosis of patients and provided new insights for individualized treatment of patients with $\mathrm{CC}$.

\section{Method}

\section{Acquisition of mRNA expression dataset}

The workflow of the present study is displayed as Fig. 1. We extracted the mRNA expression profiles of $306 \mathrm{CC}$ samples and 13 normal samples from the UCSC database (http://xena.ucsc.edu/) (Supplementary Table 1). For UCSC dataset, RNA-seq data (FPKM values) were normalized into log2 $(\mathrm{FPKM}+1)$. Then, patients with OS less than 30 days were excluded, and $273 \mathrm{CC}$ patients were included. The clinical information of $\mathrm{CC}$ patients were collected which included age, grade, American Joint Committee on Cancer (AJCC) stage, $\mathrm{T}$ classification, $\mathrm{N}$ classification, $\mathrm{M}$ classification, and OS (Supplementary Table 2).

\section{Identification and analysis of differentially expressed glycolysis-related genes (DEGRGs)}

To obtain the cancer-related genes, we compared the expression levels of 16,208 mRNAs between CC and normal tissues using the limma package. A gene with false discovery rate (FDR) $<0.05$ and $|\log \mathrm{FC}|>1$ was defined as the DEG. Then, a list of GRGs (GO_ GLYCOLYTIC_PROCESS, HALLMARK_GLYCOLYSIS, KEGG_GLYCOLYSIS_GLUCONEOGENESIS, RE ACTOME_GLYCOLYSIS, and BIOCARTA_GLYCOLYSIS_PATHWAY) was downloaded from the Molecular Signatures Database v5.1 (MSigDB). The Venn diagram was to identify the DEGRGs by combining GRGs and DEGs. Furthermore, to understand the potential function and related pathway of DEGR Gs, Gene Ontology (GO) and Kyoto Encyclopedia of Genes and Genomes (KEGG) pathway enrichment analyses were carried out by the "clusterprofiler" package.

\section{Construction and validation of the prognostic risk score signature}

Previous researches indicated that GRGs have potential prognostic value for cancer patients, but the role in $\mathrm{CC}$ patients remains unclear. Therefore, the survival analyses of GRGs in CC patients were performed in the present 


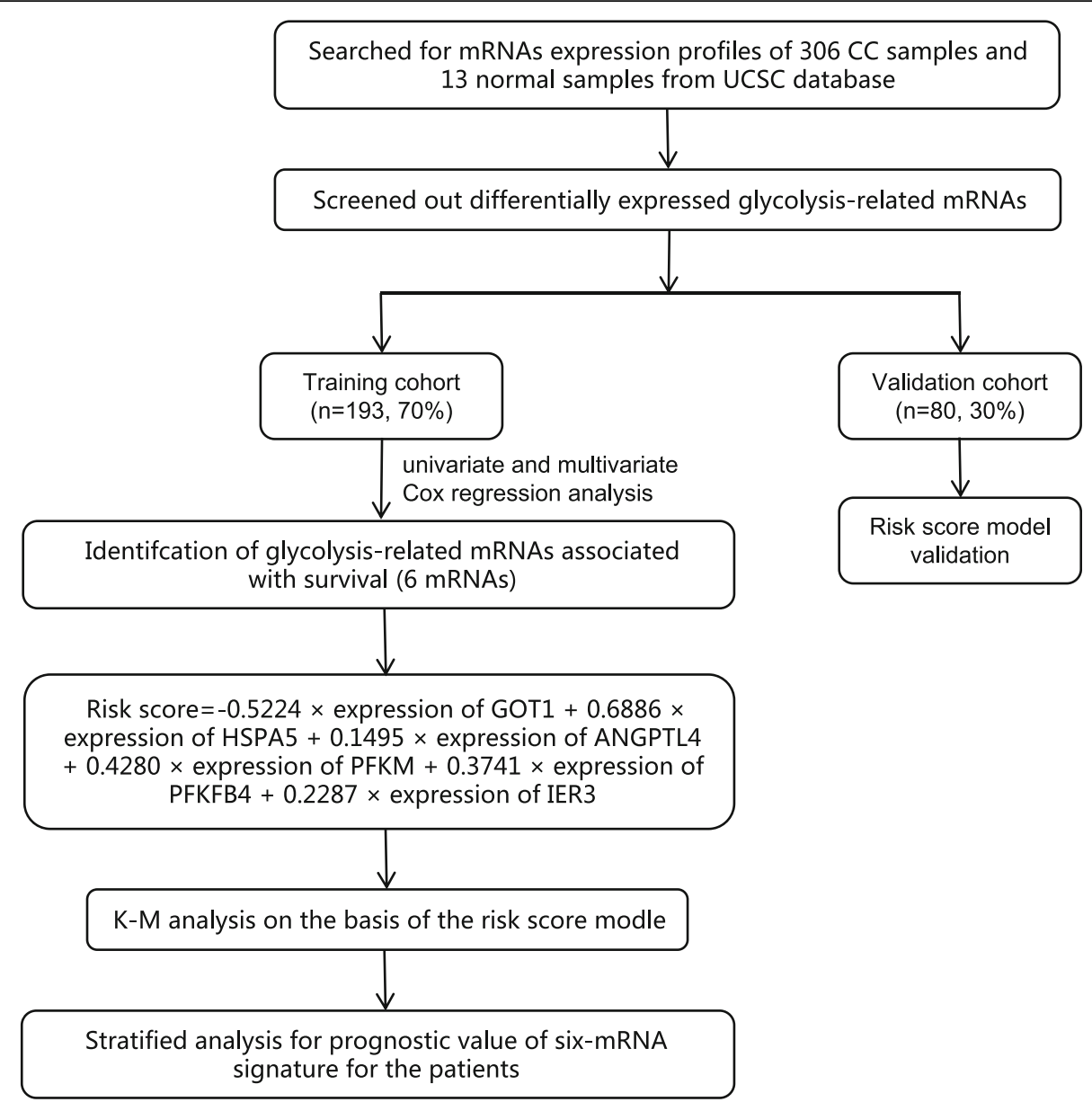

Fig. 1 Flow chart of the bioinformatic analysis

study. First, according to the ratio of 7: 3, all CC patients were randomly divided into training cohort (193 patients) and validation cohort (80 patients) (Table 1). In our research, the prognostic signature was developed in the training cohort and validated in the validation cohort. To identify the prognostic value of DEGRGs in CC patients, we performed univariate Cox regression analysis to confirm OS-related DEGRGs in training cohort. Then, based on the OS-related DEGRGs, a stepwise model selection by the Akaike information criterion (AIC) was used to avoid overfitting, and significant genes were incorporated into the multivariate Cox analysis to construct the GRG-based prognostic signature. By linear combination of the expression values of the selected genes weighted with the regression coefficients of the multivariate Cox regression analysis, the prognostic risk score model was established as follows:

$$
\text { Risk Score }=\sum_{i=0}^{n} \beta_{i} * \mathrm{G}_{i}
$$

( $\beta_{i}$ is the coefficient of the gene; $i$ in multivariate Cox analysis; $\mathrm{G}_{i}$ represents the expression value of gene $i ; n$ is the number of genes in the signature).

The time-dependent receiver operating characteristic (ROC) curve and area under the curve (AUC) value were used to evaluate the discrimination of the prognostic model. Additionally, the optimal cutoff value of risk score was determined by performing X-tile software, and patients were divided into low- and high-risk groups. Kaplan-Meier (K-M) survival curve and the log-rank test were adopted to compare the prognosis between two groups.

To further study the prognostic value of GRG signature in an independent cohort, the risk score of each patient in the validation cohort. Time-dependent ROC curve, AUC value, and K-M survival curve were used to show the prognostic ability of GRG signature in the validation cohort.

Development of a clinical-GRG nomogram for CC patients As reported in previous studies, several clinical variables were confirmed as prognostic variables, such as AJCC, T classification, $\mathrm{N}$ classification and $\mathrm{M}$ classification. 
Table 1 Clinicopathological parameters of patients with CC in the training cohort and validation cohort

\begin{tabular}{|c|c|c|c|}
\hline Clinical characteristic & $\begin{array}{l}\text { Total } \\
(N=273) \\
\mathrm{N}(\%)\end{array}$ & $\begin{array}{l}\text { Training cohort } \\
(N=193) \\
\mathrm{N}(\%)\end{array}$ & $\begin{array}{l}\text { Validation cohort } \\
(N=80) \\
\mathrm{N}(\%)\end{array}$ \\
\hline \multicolumn{4}{|l|}{ Age } \\
\hline$<50$ & $171(62.6)$ & $124(64.2)$ & $47(58.8)$ \\
\hline$>50$ & $102(37.4)$ & 69 (35.8) & $33(41.2)$ \\
\hline \multicolumn{4}{|l|}{ Grade } \\
\hline G1-G2 & $142(52)$ & $103(53.4)$ & $39(48.8)$ \\
\hline G3-G4 & $105(38.5)$ & $72(37.3)$ & $33(41.2)$ \\
\hline NA & $26(9.5)$ & $18(9.3)$ & $8(10)$ \\
\hline \multicolumn{4}{|l|}{ T classification } \\
\hline $\mathrm{T} 1-\mathrm{T} 2$ & $192(70.3)$ & $139(72)$ & $53(66.3)$ \\
\hline T3-T4 & $26(9.5)$ & $17(8.8)$ & $9(11.2)$ \\
\hline NA & $55(20.1)$ & $37(19.2)$ & $18(22.5)$ \\
\hline \multicolumn{4}{|l|}{ N classification } \\
\hline No & $118(43.2)$ & $92(47.7)$ & $26(32.5)$ \\
\hline N1 & $53(19.4)$ & $32(16.5)$ & $21(26.3)$ \\
\hline NA & $102(37.4)$ & 69 (35.8) & $33(41.2)$ \\
\hline \multicolumn{4}{|l|}{ M classification } \\
\hline Mo & $101(37)$ & 69 (35.8) & $32(40)$ \\
\hline M1 & $10(3.7)$ & $8(4.1)$ & $2(2.5)$ \\
\hline NA & $162(59.3)$ & $116(60.1)$ & $46(57.5)$ \\
\hline \multicolumn{4}{|l|}{ AJCC stage } \\
\hline$|-| \mid$ & 209 (76.6) & $150(77.7)$ & $59(73.8)$ \\
\hline III-IV & $58(21.2)$ & 38 (19.7) & $20(25)$ \\
\hline NA & $6(2.2)$ & $5(2.6)$ & $1(1.2)$ \\
\hline
\end{tabular}

Abbreviations: $T$ Tumor, $N$ Node (regional lymph node), $M$ Metastasis

Therefore, to develop a comprehensive prognostic model for CC patients, we firstly studied the prognostic value of clinical data, including age, grade, ATCC stage and TNM classification in CC patients by univariate Cox analysis. Moreover, the OS-related clinical data and GRG signature were incorporated into the multivariate Cox analysis and the independent variables were used to develop a nomogram. Concordance-index(C-index) and calibration curve were used to evaluate the nomogram.

\section{Specimens sources}

Ten cases of CC and 10 cases of normal cervical tissue were obtained from the Department of gynecology and obstetrics, the First Affiliated Hospital of Wenzhou Medical University. Informed consent was provided by all patients. This study was approved by the ethics committee of the First Affiliated Hospital of Wenzhou Medical University. All tissues were pathologically diagnosed and stored in $-80^{\circ} \mathrm{C}$ for preservation.

\section{Conducting quantitative real time (qRT)-PCR on tissues}

Total RNA was refined from clinical specimens by TRI$\mathrm{zol}^{\circ}$ reagent (Vazyme, Nanjing, China), then, following the manufacturer's protocol, a PrimeScript RT reagent kit (Vazyme) was used to reverse transcription. qRTPCR was performed to evaluate the DEGRGs expression in different specimens using SYBR-Green Premix (Vazyme) with specifc PCR primers shown in Supplementary Table 3. With GAPDH as the internal control, and the $\mathrm{Ct}$ method $(2-\Delta \Delta \mathrm{Ct})$ was used to normalize the relative genes expression values.

\section{Correlation analysis between immune cell infiltration and glycolysis}

We adopted CIBERSORT [18], which is widely used to describe the immune cell composition of the gene expression profiles to explain the 22 immune cell subtypes. The global $P$ value of each sample deconvolution was determined by CIBERSORT, and samples with CIBERSORT $P<0.05$ was selected for further study. Thus, we included a total of 184 patients from 193 patients. To 
determine the relationship between immune cell infiltration and glycolysis, we divided 184 patients into highand low-risk groups based on the prognostic risk score and compared the differences in immune cells between the two groups and displayed the results by heatmap and violin plot. Furthermore, we conducted univariate Cox regression, LASSO and multivariate Cox regression analyses to examine whether the risk score and immune cells can be used as independent factors to predict the prognosis of CC patients.

\section{Results}

\section{Preliminary screening of glycolysis-related mRNA}

We obtained the mRNA expression profiles from the UCSC database, including 306 tumour samples and 13 normal samples. By comparing tumour and normal tissue samples, we screened 4772 differentially expressed mRNAs from 16,208 mRNAs. Then, Venn diagram software was applied to identify the DEGRGs in the 4772 differentially expressed mRNAs and 5 glycolytic gene sets, and the results showed that 96 DEGRGs were detected (Fig. 2).

To further analyse the relationship between these genes and glycolysis, we carried out GO analysis and KEGG pathway enrichment analysis on these genes. As a result, we found in GO analysis that the primary enriched terms were ADP metabolic process, pyruvate metabolic process and glycolytic process (Fig. 3a). For KEGG pathway enrichment analysis, glycolysis/gluconeogenesis, fructose and mannose metabolism and carbon metabolism were the most enriched. Based on these results, it has been shown that the genes we selected are indeed related to glycolysis (Fig. 3b).

\section{Identification of DEGRGs associated with survival and visualization of the DEGRG status}

We analysed 96 mRNAs selected above to screen out survival-related mRNAs in training cohort by univariate Cox regression analysis and obtained 19 mRNAs with $P<0.05$ (Fig. 4a). Next, we performed a stepwise model selection by the AIC, and six mRNAs (HSPA5, ANGP TL4, PFKM, GOT1, IER3 and PFKFB4) were obtained. Finally, multivariate Cox analysis was carried out to further identify the relationship of selected mRNAs with CC patients' prognosis and obtained the coefficients. The filtered mRNAs were classified into the protective type (GOT1), whose $\mathrm{HR}<1$ was related to better prognosis, and the risk type (HSPA5, ANGPTL4, PFKM, IER3 and PFKFB4), whose HR > 1 was related to poor prognosis (Table 2).

In addition, to examine the DEGRG status of the whole genome, we visualized the data by using Circos plots [19], which are shown in Fig. 4b. The outer layer includes chromosomes and 96 DEGRGs. The middle four layers, from the outside to the inside, are the average expression values of DEGRGs in normal tissues, the average expression values of DEGRGs in tumour tissues, the $\log \mathrm{FC}$ of the difference analysis, and the 19 meaningful prognostic DEGRGs obtained by univariate Cox regression analysis in the training cohort. The inner layer is the protein-protein interaction (PPI) network of the DEGRGs. In addition, 0.4 was defined as the minimum required interaction

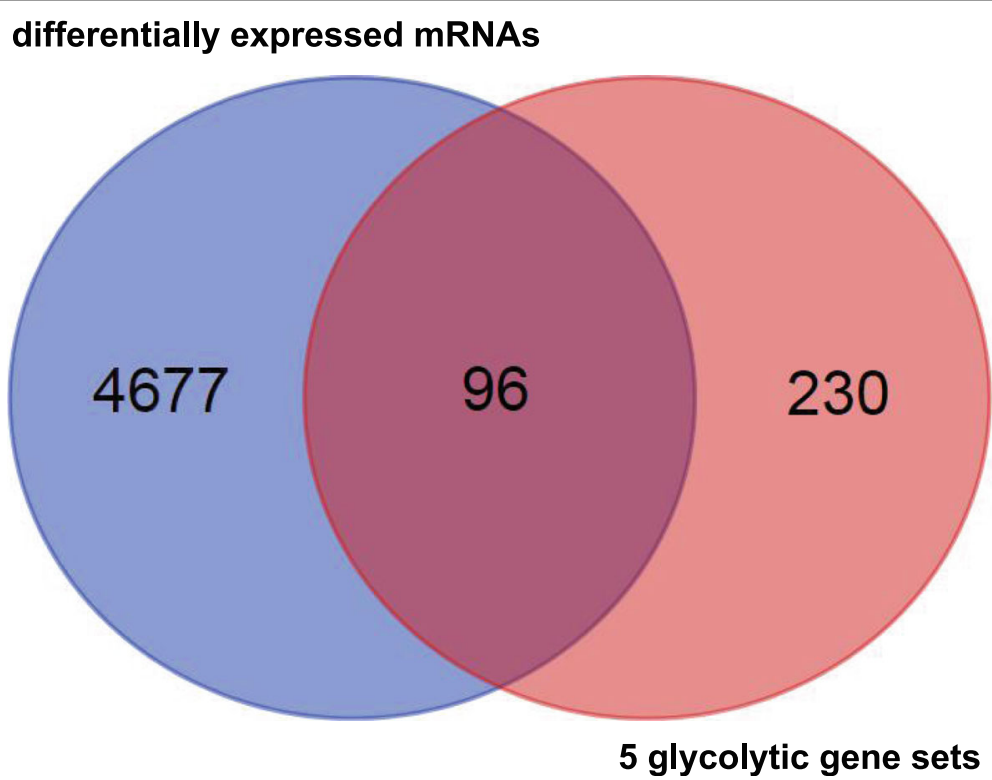

Fig. 2 Identification of 96 DEGRGs in the differentially expressed mRNAs and 5 glycolytic gene sets (GO_GLYCOLYTIC_PROCESS, HALLMARK _GLYCOLYSIS, KEGG_GLYCOLYSIS_GLUCONEOGENESIS, REACTOME_GLYCOLYSIS, and BIOCARTA_GLYCOLYSIS_PATHWAY) 

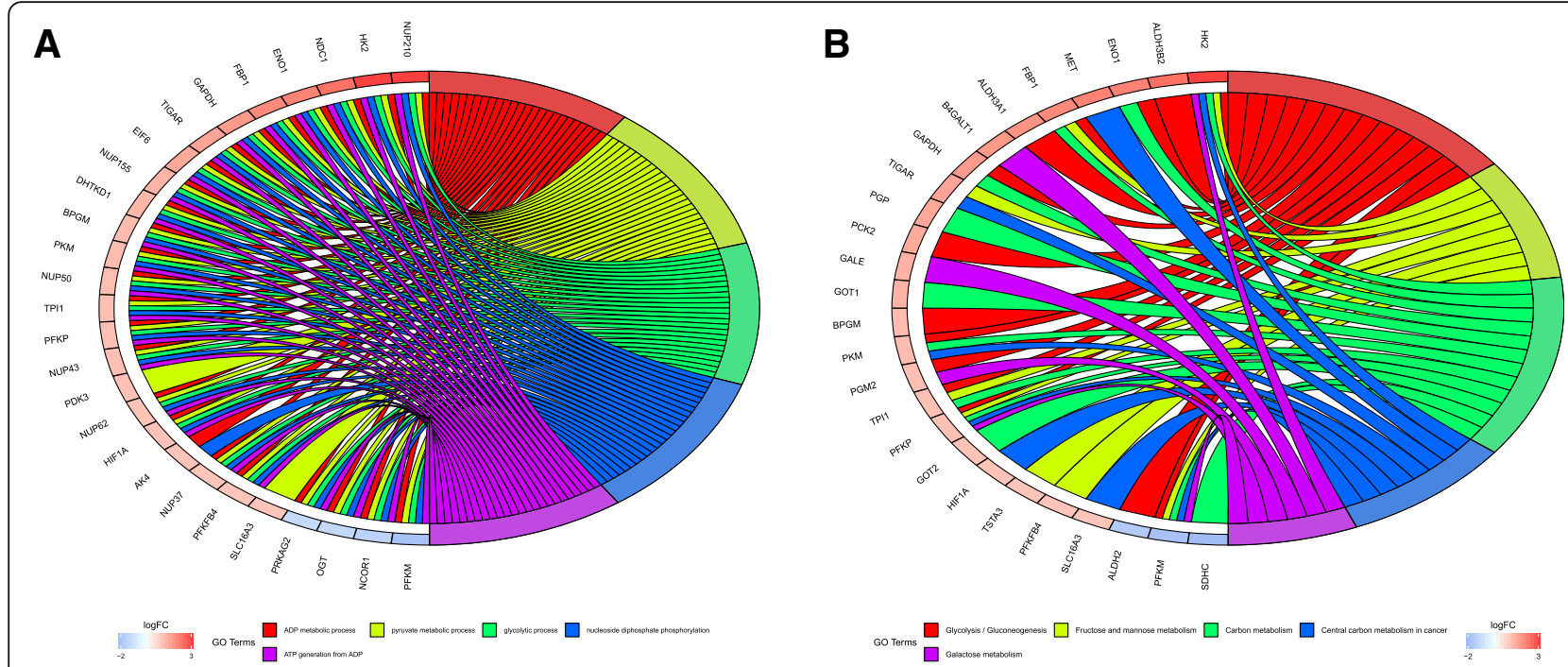

Fig. 3 GO and KEGG pathway enrichment analysis of DEGRGs. a GO analysis. b KEGG pathway enrichment analysis. The right shows significantly enriched GO or KEGG terms, each bar on the left represents a gene and the depth of the color represents the logFC value of the gene. The intermediate line represents the connections between genes and GO or KEGG terms

score, and between 0.4 and 0.9 is displayed in blue, while greater than 0.9 is displayed in red.

\section{Constructing and validating a six-mRNA signature to predict patient prognosis}

Using the linear combination of the expression value of the selected genes and the regression coefficient of multivariate Cox regression analysis, the following predictive risk scoring model was established: risk score $=-0.5224 \times$ expression of GOT1 $+0.6886 \times$ expression of HSPA $5+$ $0.1495 \times$ expression of ANGPTL4 $+0.4280 \times$ expression of PFKM $+0.3741 \times$ expression of PFKFB4 $+0.2287 \times$ expression of IER3. Based on the prognosis risk score, 193 patients were divided into high- and low-risk groups by using optimal risk score cutoff identified by X-tile as the boundary value (Fig. 5a), and the respective survival status of 193

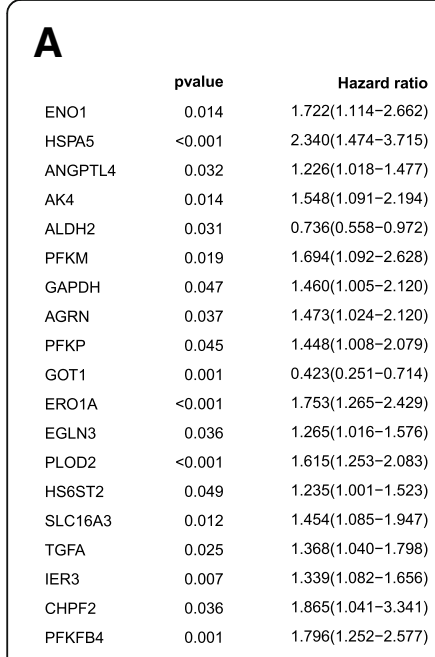

Fig. 4 The results of univariate Cox analysis and circos analysis of DEGRG status in the whole genome. a Nineteen DEGRGs associated with survival. b The outermost layer shows 96 DEGRGs and their location in the whole genome, the middle part from the outside to the inside shows the expression of these genes in normal and tumour tissues, and the 19 DEGRGs associated with prognosis obtained by univariate Cox regression analysis in the training cohort. The inner layer is the PPI network. The medium confidence of the minimum required interaction score is $0.4,<0.9$ is blue, and $>0.9$ is red

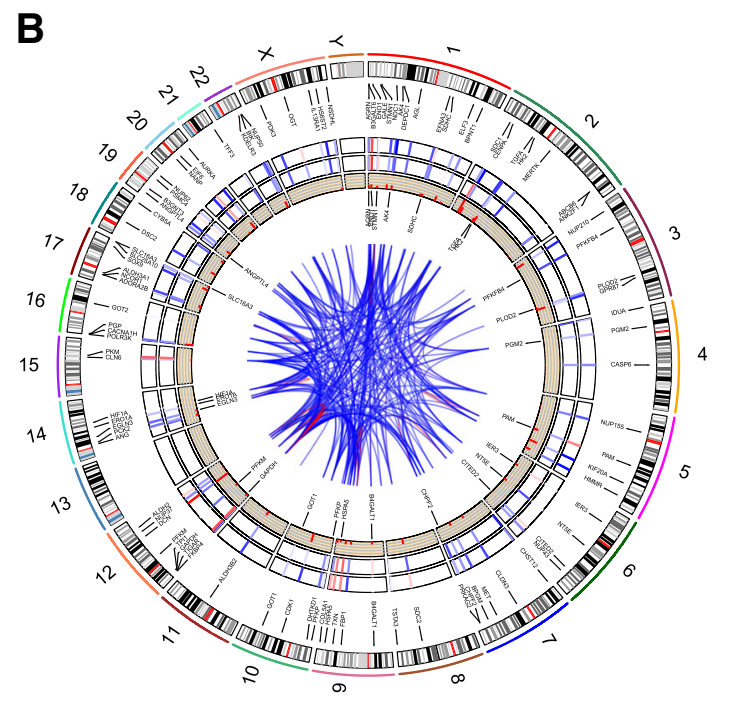

B

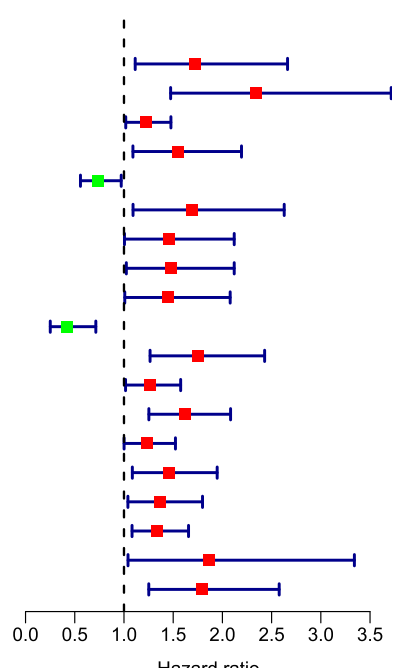


Table 2 Information on six prognostic mRNAs significantly related to overall survival in patients with CC

\begin{tabular}{lllll}
\hline mRNA & $\mathbf{B}$ (Cox) & HR & 95\%Cl of HR & P \\
\hline HSPA5 & 0.6886 & 1.99 & $1.14-3.48$ & 0.015 \\
ANGPTL4 & 0.1495 & 1.16 & $0.96-1.41$ & 0.125 \\
PFKM & 0.428 & 1.53 & $0.95-2.48$ & 0.081 \\
GOT1 & -0.5224 & 0.59 & $0.34-1.05$ & 0.072 \\
IER3 & 0.2287 & 1.26 & $0.99-1.60$ & 0.064 \\
PFKFB4 & 0.3741 & 1.45 & $0.97-2.18$ & 0.070 \\
\hline
\end{tabular}

patients was presented (Fig. 5b). The K-M analysis showed that compared to the high-risk group, the OS in the lowrisk group was dramatically better $(p<0.0001$; Fig. $5 c)$. The AUCs for 1-, 3- and 5-year OS were 0.791, 0.731 and 0.782, respectively (Fig. 5d), suggesting that the six-mRNA signature has excellent diagnostic significance for prognosis prediction. Additionally, we generated a heatmap to exhibit the expression profiles of the six mRNAs, from which we can see that, compared with the low-risk group, the expression level of risk-type mRNAs (HSPA5, ANGPTL4, PFKM, IER3 and PFKFB4) of the high-risk group was apparently higher, while the expression level of the protective-type mRNA GOT1 was opposite (Fig. 5e).

Next, we validated the predictive ability of the six-mRNA signature in the validation cohort. Eighty patients were divided into high- and low-risk groups by using the same optimal risk score cutoff which were used in the training cohort (Fig. 6a), and the respective survival status of 80 patients was presented (Fig. 6b). And the K-M analysis showed that the patients in the low-risk group had significantly better OS $(P=0.021)$ (Fig. 6c), which AUC values were $0.664,0.635$ and 0.661 for 1-, 3- and 5-year, respectively (Fig. 6d).

The risk score constructed by the six-mRNA signature is an independent prognostic indicator

To evaluate whether the six-mRNA signature was an independent prognostic indicator, we performed univariate
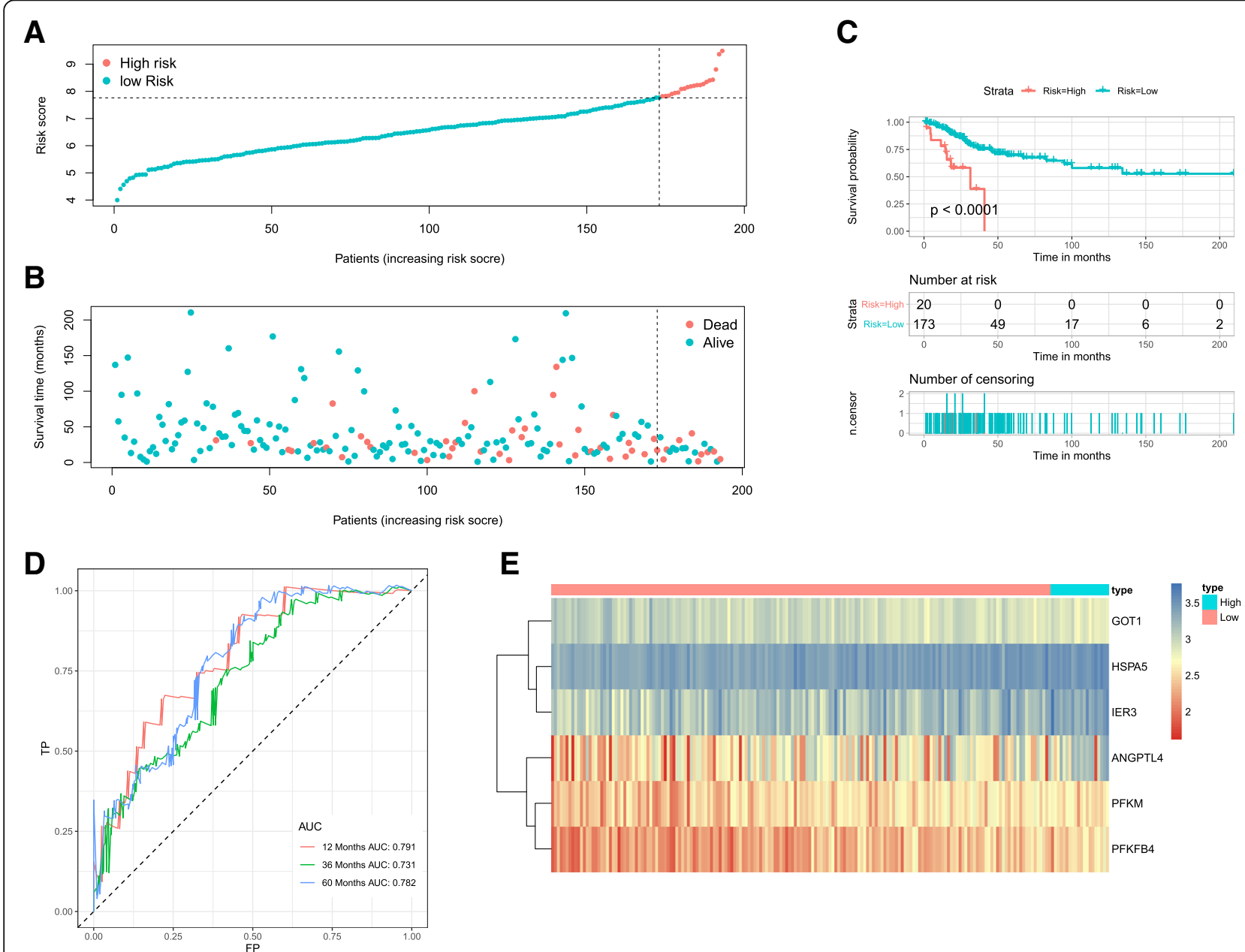

Fig. 5 The six-mRNA signature associated with risk score predicts OS in the training cohort. a mRNA risk score distribution. b Survival days of patients. c K-M curve to test the predictive effect of the gene signature in the training cohort. $\mathbf{d}$ ROC curve analysis to evaluate the sensitivity and specificity of the gene signature. e A heatmap of the expression profile of six mRNAs 

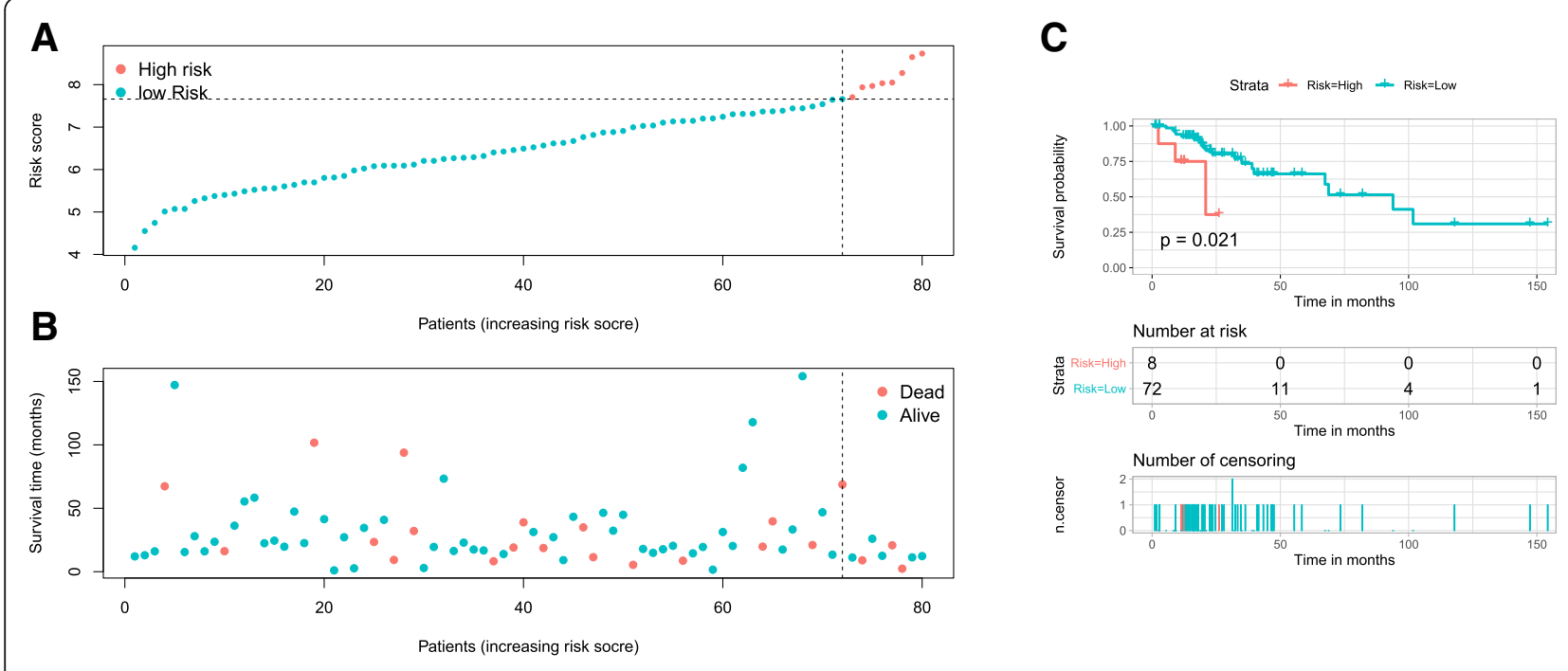

\section{$\mathbf{E}$}
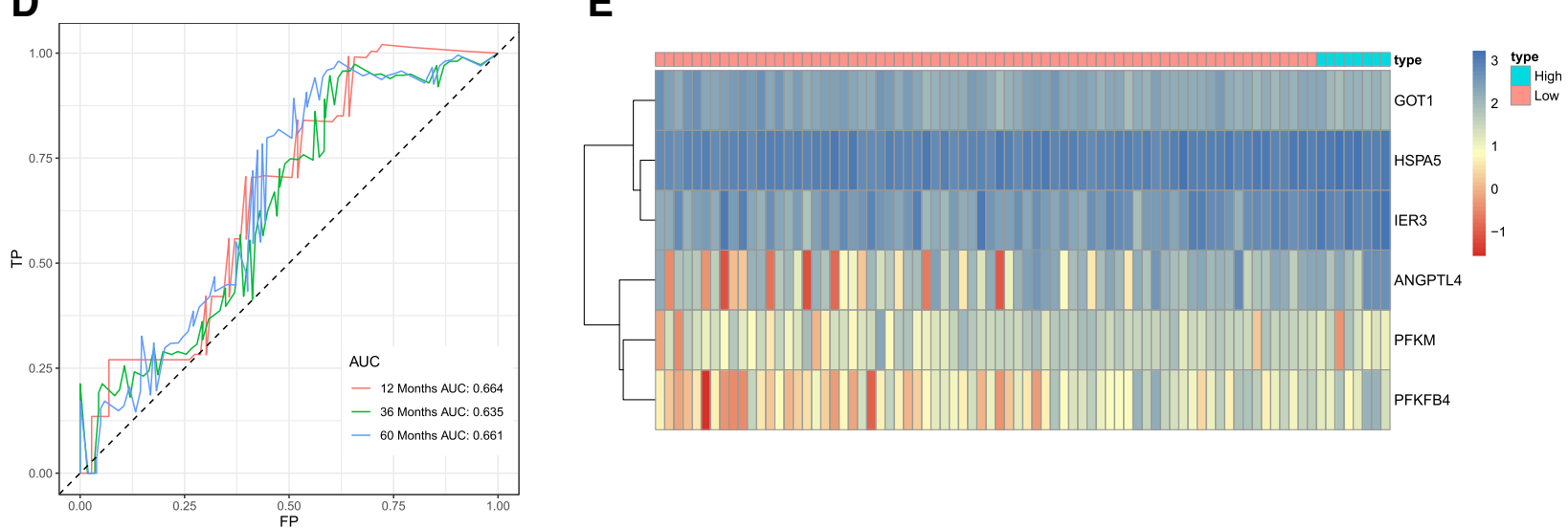

Fig. 6 Validation of the six-mRNA signature in the validation cohort. a mRNA risk score distribution. $\mathbf{b}$ Survival days of patients. $\mathbf{c}$ K-M curve to test the predictive effect of the gene signature in the validation cohort. $\mathbf{d}$ ROC curves of the gene signature. e A heatmap of the expression profile of six mRNAs

and multivariate Cox regression analyses to compare the prognostic values of risk score with other clinical characteristics. We included a total of 193 patients, and among these patients, $124(64.2 \%)$ were younger than 50 years old, 69 (35.8\%) were older than 50 years old. Among 193 patients, $103(53.4 \%)$ had grade $1-2$ tumours, and 72 (37.3\%) had grade 3-4 tumours, 139 (72.0\%) had T1-T2 tumours, 17 (8.8\%) had T3-T4 tumours. Furthermore, among these patients, 92 (47.6\%) had no lymph node metastasis, 32 (16.5\%) had lymph node metastasis, 69 (35.7\%) had no distant metastasis, and $8(4.1 \%)$ had distant metastasis. Additionally, among these patients, 150 (77.7\%) had stage I-II disease, and 38 (19.7\%) had stage III-IV disease. In all the above data, except for age and grade, AJCC stage $(\mathrm{HR}=2.78,95 \%$ CI $1.55-4.98, p<0.001)$, T classification $(\mathrm{HR}=5.15,95 \%$ CI $2.55-10.42, p<0.001), \mathrm{M}$ classification $(\mathrm{HR}=4.70,95 \% \quad \mathrm{CI} 1.53-14.50, \quad p=0.007)$ and $\mathrm{N}$ classification $(\mathrm{HR}=4.18,95 \%$ CI 1.83-9.53 $p<0.001)$ showed significant differences in the univariate Cox regression analysis, which can be used as independent prognostic factors (Table 3). In the next multivariate Cox regression analysis, only the $\mathrm{N}$ classification showed significant differences ( $\mathrm{HR}=14.80,95 \%$ CI 3.44-63.76, $p<$ 0.001) (Table 3). However, regardless of univariate or multivariate Cox regression analysis, the risk score showed significant prognostic value $(\mathrm{HR}=2.72,95 \% \mathrm{CI} 1.92-3.85$, $p<0.001 ; \quad \mathrm{HR}=2.57,95 \%$ CI $1.47-4.49, \quad p=0.002)$ (Table 3). Based on these results above, we constructed a nomogram prediction model combined the risk score with $\mathrm{N}$ classification to predict CC patients' OS (Fig. 7a), which $\mathrm{C}$ index is 0.83 . The calibration plot showed that in the nomogram, the predicted values of OS at 1, 3 and 5 years for $\mathrm{CC}$ patients have a good correlation with the actual values (Fig. 7b-d). 
Table 3 Univariable and multivariable Cox regression analysis for each clinical feature

\begin{tabular}{|c|c|c|c|c|c|c|}
\hline \multirow[t]{2}{*}{ Clinical feature } & \multicolumn{3}{|c|}{ Univariate analysis } & \multicolumn{3}{|c|}{ Multivariate analysis } \\
\hline & $\mathrm{HR}$ & $\begin{array}{l}95 \% \mathrm{Cl} \text { of } \\
\mathrm{HR}\end{array}$ & $P$ value & $\mathrm{HR}$ & $95 \% \mathrm{Cl}$ of $\mathrm{HR}$ & $P$ value \\
\hline Risk score & 2.72 & $1.92-3.85$ & $<0.001$ & 2.57 & $1.47-4.49$ & 0.002 \\
\hline Age $(\leq 50 />50)$ & 1.37 & $0.78-2.41$ & 0.276 & - & - & - \\
\hline Grade (G1-G2/G3-G4) & 1.03 & $0.55-1.93$ & 0.925 & - & - & - \\
\hline T (T1-T2/T3-T4) & 5.15 & $2.55-10.42$ & $<0.001$ & - & - & - \\
\hline M (M0/M1) & 4.70 & $1.53-14.50$ & 0.007 & - & - & - \\
\hline $\mathrm{N}(\mathrm{N} 0 / \mathrm{N} 1)$ & 4.18 & $1.83-9.53$ & $<0.001$ & 14.80 & $3.44-63.76$ & $<0.001$ \\
\hline AJCC stage (I-II/III-IV) & 2.78 & $1.55-4.98$ & $<0.001$ & - & - & - \\
\hline
\end{tabular}

Abbreviations: $T$ Tumor, $N$ Node (regional lymph node), $M$ Metastasis, HR Hazard ratio, 95\% Cl 95\%Confidence Interval

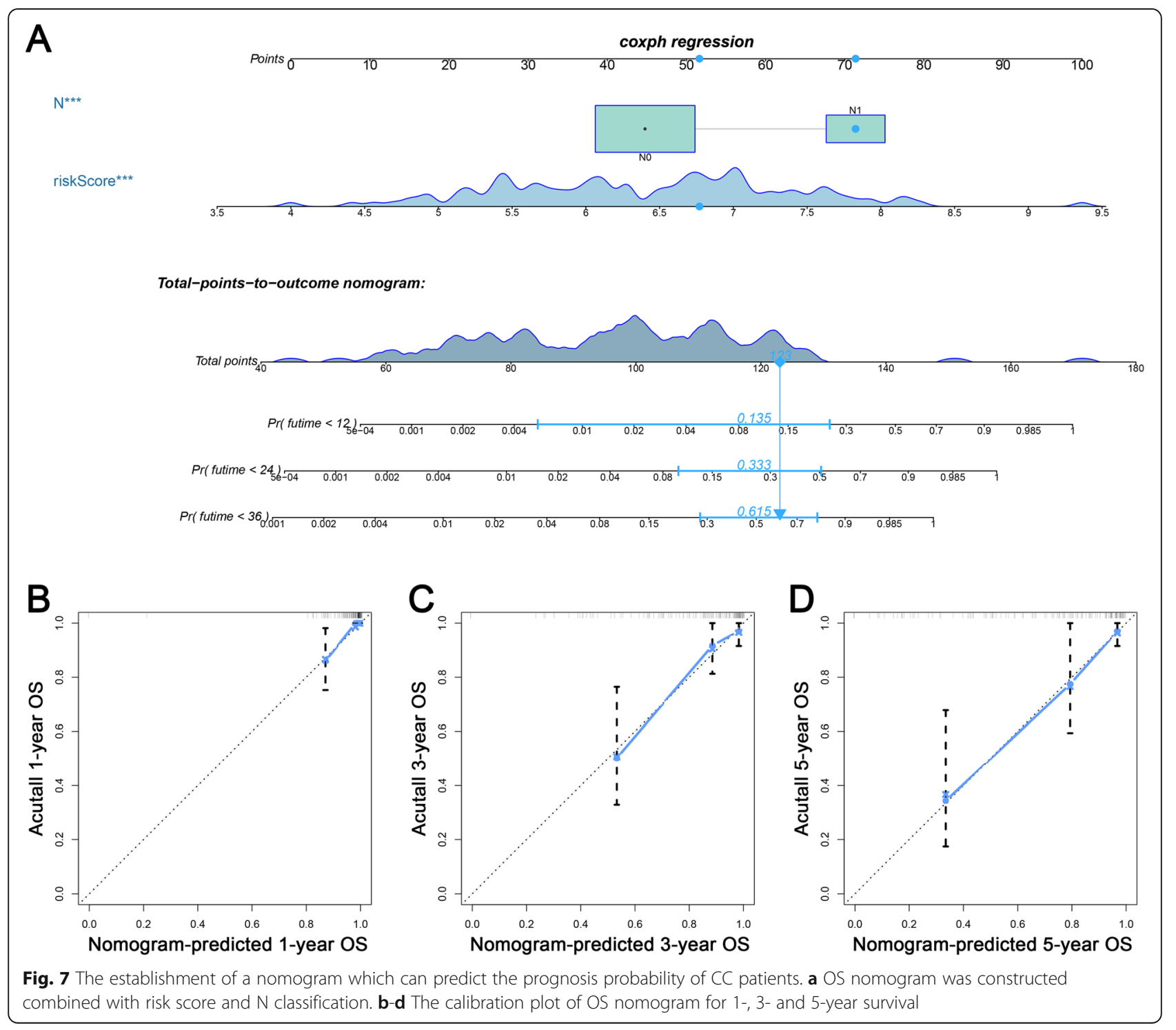


Next, stratified analysis was performed to further validate whether the six-mRNA signature can be used as an independent prognostic factor, according to the grade, AJCC, T classification and age. First, we classified patients into the grade 1-2 dataset and grade 3-4 dataset, and the patients in the datasets were divided into high- and lowrisk groups, respectively. As a result, we found that there were significant differences in OS between these two groups, and the OS of the low-risk group was obviously better (Fig. 8a). Next, we did the same thing for the other clinicopathological features, and we found that the OS of the high-risk group was significantly worse than that of the low-risk group regardless of the AJCC stage (stage I-II or stage III-IV) (Fig. 8b), T classification (T0 or T1) (Fig. 8c) and age (younger than 50 or older than 50) (Fig. 8d), further confirming the reliability of our analysis.

\section{Validating the overexpression of the six mRNAs in CC tissues by qRT-PCR}

We validated the expression level of six mRNAs in 10 $\mathrm{CC}$ tissues and 10 normal cervical tissues by using qRTPCR. The results showed that compared with the normal cervial tissuses, the expression level of HSPA5, ANGP TL4, PFKM, GOT1, IER3 and PFKFB4 were significantly higher in the $\mathrm{CC}$ tissue (Fig. 9), making the bioinformatics analysis result much more reliable and precise.

\section{The relationship between immune cell infiltration and glycolysis}

We analysed a total of 184 patients' immune cell composition from their gene expression profiles. Additionally, we divided these patients into two groups, and we can see that the expression levels of CD8 $\mathrm{T}$ cells, regulatory $\mathrm{T}$ cells (Tregs) and resting mast cells were significantly higher in the low-risk group compared to the high-risk group; however, in the high-risk group, the expression levels of neutrophils, M0 macrophages, activated mast cells and resting CD4 memory T cells were dramatically higher (Fig. 10).

In addition, we analyzed the relationship between immune cells and prognosis of $\mathrm{CC}$ patients. In the univariate Cox regression analysis, CD8 $\mathrm{T}$ cells, resting CD4 memory T cells, M0 macrophages, M2 macrophages, resting mast cells, activated mast cells and Neutrophils all showed significant differences with $p<0.05$. Among these immune cells, the HR of CD8 $\mathrm{T}$ cells, M2 macrophages and resting mast cells were less than 1, which associated with better prognosis. In the next multivariate Cox regression analysis, only activated mast cells showed significant differences with $p<0.05$. And both in univariate or multivariate Cox regression analysis, the risk score showed significant prognostic value $(p<0.001)$ (Table 4$)$.

\section{Discussion}

In recent years, increasing attention has been paid to the relationship between energy metabolism and tumours, among which the Warburg effect is a notable feature of the energy metabolism of tumour cells. Despite the low efficiency of glycolysis, tumour cells are still active in glycolysis, which can produce more energy and various metabolites in a short time so that tumour cells can
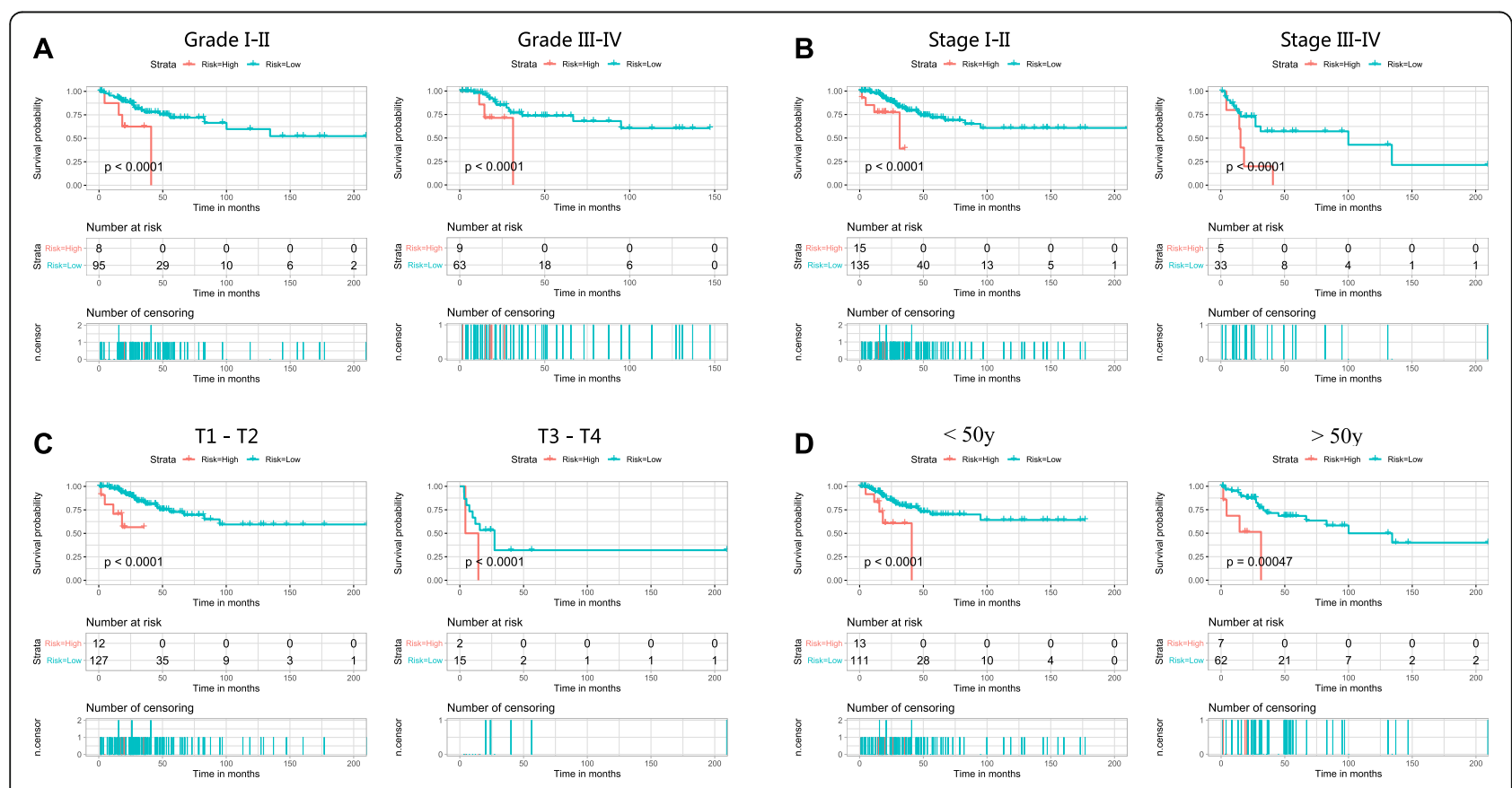

Fig. 8 Stratified analysis for the prognostic value of the six-mRNA signature for patients. a Grade, b AJCC stage, c T classification, $\mathbf{d}$ Age 


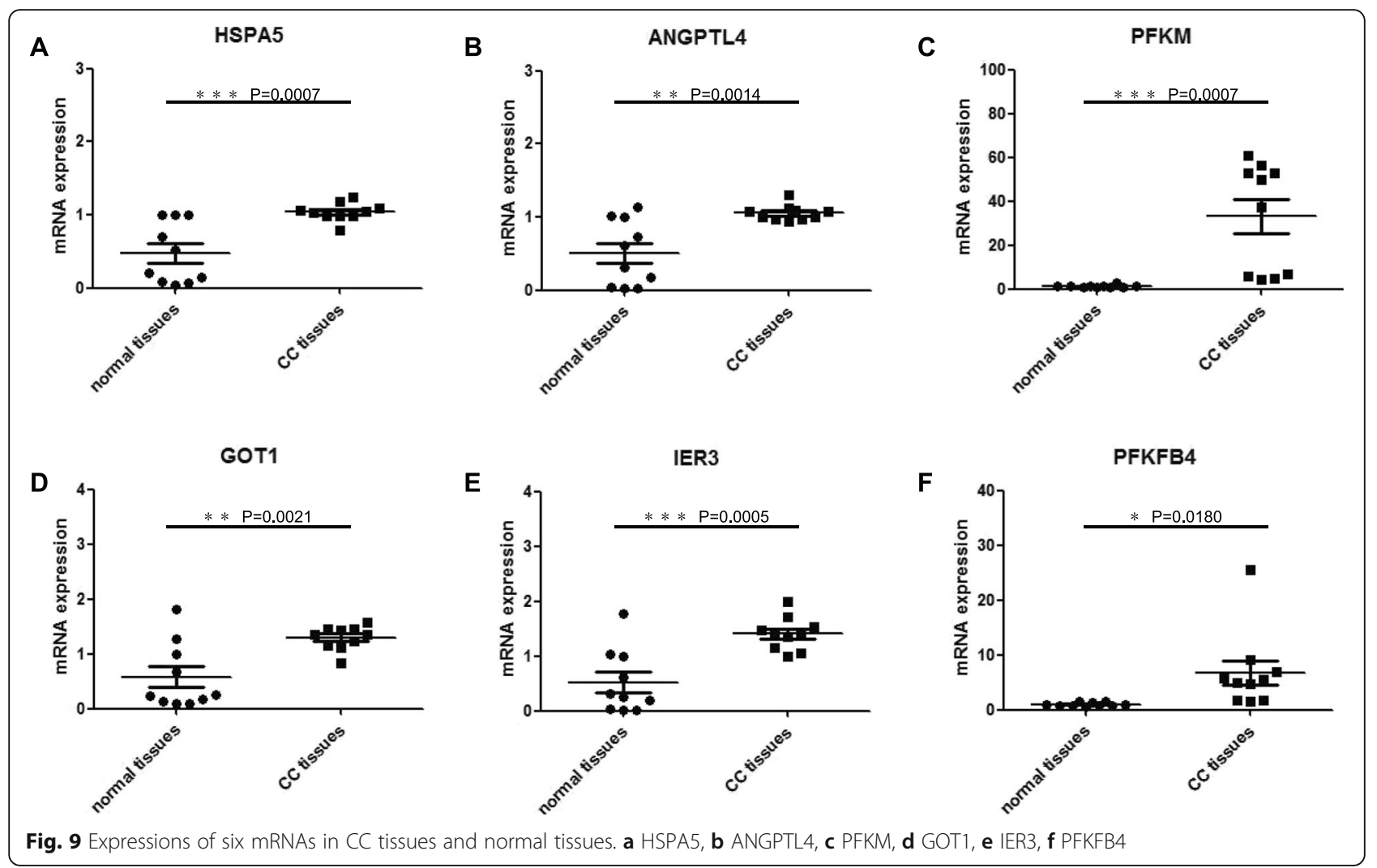

benefit from glycolysis [6]. More importantly, the rapid growth of tumours is often accompanied by hypoxia, and hypoxia inducible factor (HIF), as the key regulator of glycolysis, can solve the nutritional needs of tumour cells by inducing the expression of glucose and amino acid transporters (glucose transporter type 1 (GLUT1)) and L-type amino acid transporter 1 (LAT1) to further promote the progression of cancer $[20,21]$. There have been many studies on the relationship between glycolysis and tumours, but the relationship between GRGs and the prognosis of patients with $\mathrm{CC}$ is still very limited. Because a single gene biomarker cannot provide a strong prediction effect, a more accurate and reliable gene signature was used to predict the clinical outcomes of patients. For instance, a glycolysis-related nine-gene signature was used to predict the survival of patients with endometrial cancer [17]. A tumour microenvironmentrelated nine-gene signature was used to predict overall survival with ovarian cancer [22]. Moreover, an autophagy-related five-gene signature was developed for

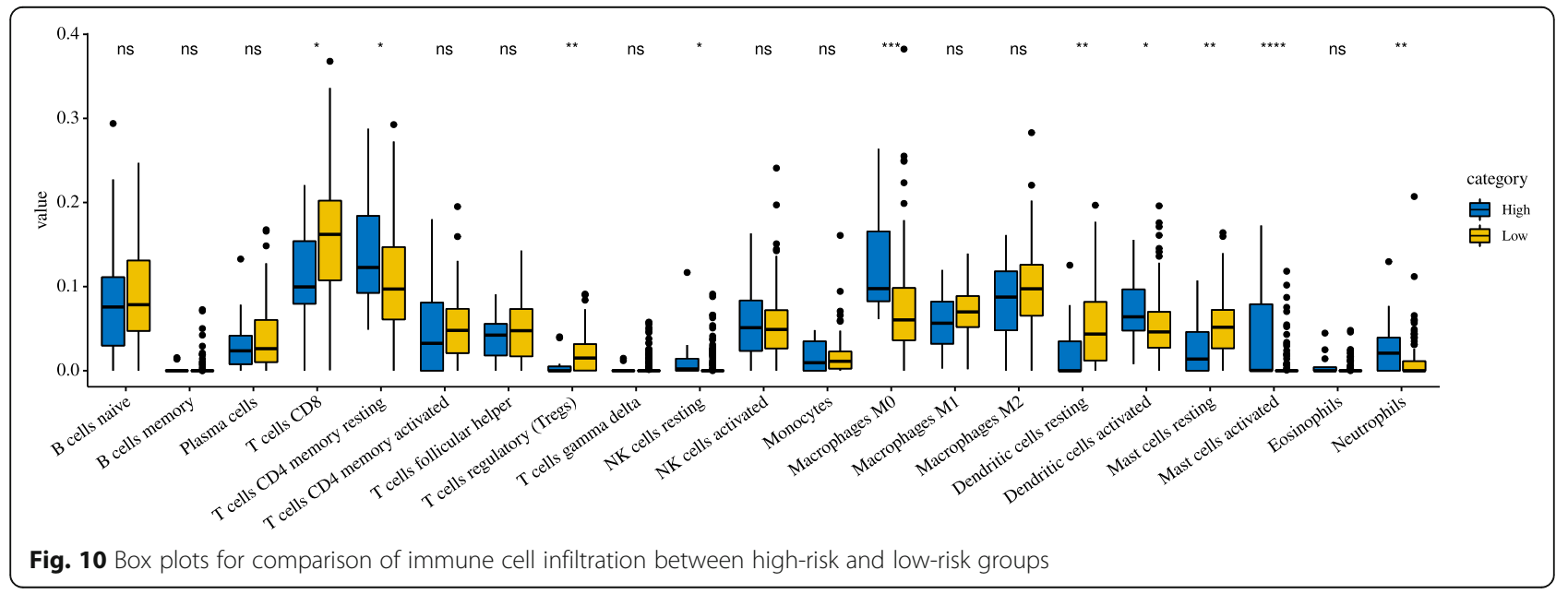


Table 4 Univariable and multivariable Cox regression analysis for immune cells

\begin{tabular}{|c|c|c|c|c|c|c|}
\hline \multirow[t]{2}{*}{ Immune cells } & \multicolumn{3}{|c|}{ Univariate analysis } & \multicolumn{3}{|c|}{ Multivariate analysis } \\
\hline & HR & $95 \% \mathrm{Cl}$ of $\mathrm{HR}$ & $P$ value & HR & $95 \% \mathrm{Cl}$ of $\mathrm{HR}$ & $P$ value \\
\hline Risk Score & 2.58 & $1.80-3.71$ & $<0.001$ & 2.11 & $1.43-3.11$ & $<0.001$ \\
\hline T cells CD8 & 0.002 & $3.89 E-05-0.10$ & 0.002 & - & - & - \\
\hline T cells CD4 memory resting & 174.15 & 3.19-9503.14 & 0.011 & - & - & - \\
\hline Macrophages M0 & 3081.50 & $23.73-400,288.68$ & 0.001 & - & - & - \\
\hline Macrophages M2 & 0.00 & $9.46 \mathrm{E}-08-0.22$ & 0.018 & - & - & - \\
\hline Mast cells resting & 0.00 & $1.14 \mathrm{E}-10-0.05$ & 0.011 & - & - & - \\
\hline Mast cells activated & 2.43E-06 & $207,417.10-2.4 \mathrm{E}+11$ & $<0.00122925 .08$ & $6.23-8 \mathrm{E}+07$ & 0.017 & \\
\hline Neutrophils & $2.23 E+8$ & $81.22-5.28 E+10$ & 0.005 & - & - & - \\
\hline
\end{tabular}

prognosis prediction in patients with prostate cancer [23]. In this study, we used the GRG signature for the first time to predict the survival of patients with $\mathrm{CC}$ and obtained a good prediction effect.

First, we screened out the differentially expressed glycolytic mRNAs from the CC dataset in the UCSC database and selected six DEGRGs with predictive ability for the prognosis of patients with $\mathrm{CC}$ in the training cohort through univariate and multivariate Cox regression analysis $(P<0.05)$. Subsequently, by constructing the prognostic risk score model, we found that there was a significant difference in OS between the high- and lowrisk groups, and the OS of the high-risk group was evidently worse. Furthermore, the risk score model was validated in the validation cohort with good predictive ability. Finally, further verification was done through PCR, we found that the expression of these six genes in tumor tissue was indeed significantly higher than that in normal tissue.

From our analysis, we selected six DEGRGs (HSPA5, ANGPTL4, PFKM, GOT1, IER3 and PFKFB4). Heat shock protein A5 (HSPA5, also konwn as GRP78), a member of the HSP70 family, has been found to play an important role in cancer malignancy and anti-tumor therapy. And it has been reported that the high expression of HSPA5 can protect cancer cells from immune surveillance, and inhibition of its expression can promote cell apoptosis and inhibit tumor growth $[24,25]$. Angiopoietin-like4 (ANGP TL4) is highly expressed in various tumors, which is closely related to tumor growth and metastasis, such as hepatocellular carcinoma [26], breast cancer [27], head and neck squamous cell carcinoma [28] and melanoma [29]. And ANGPTL4 participates in the construction of GRG signature in lung adenocarcinoma to predict the survival and metastasis of patients [14]. In addition, previous studies have found that ANGPTL4 is significantly associated with the susceptibility of CC, is a potential risk factor [30]. Muscular phosphofructokinase (PFKM), a member of the phosphofructokinase (PFK) family, can promote the growth of muscle-infiltrating bladder cancer [31] and can be used as a new breast cancer gene [32], and its expression level can distinguish normal tissues from CC tissues [33]. 6-Phosphofructo-2-kinase/fructose-2, 6-biphosphatase 4 (PFKFB4), is a key kinase in Warburg pathway [34], has been found to be associated with a variety of cancers, including breast cancer [35], prostate cancer [36] and glioblastoma [37], promoting the progression and metastasis of cancer, and may become an effective molecular target of anti-tumor drugs. It was found that the expression of immediate early response gene 3 (IER3) was increased in advanced cancer [38, 39], but some studies have found that IER3 can also promote tumour cell apoptosis and has anti-tumour activity, such as lower expression in CC tissues [40], and increasing the expression of IER3 can enhance the sensitivity of CC cells to radiotherapy [41]. In contrast, in our study, we found that IER3 exists as a risk factor. Moreover, glutamate oxaloacetate transaminase 1 (GOT1), is a gene that encodes cytoplasmic aspartate aminotransferase and a key aspartate-producing protein. Glutamine metabolism is essential for the proliferation of cancer cells in addition to glucose. Glutamine derived glutamic acid is used to generate nonessential amino acids by GOT1 in high proliferative cells, which plays an important role in cell proliferation [42, 43]. Moreover, HIFa can inhibit the proliferation of tumor cells by inhibiting the synthesis of aspartate [44]; however, in our study, we found that GOT1 was a protective factor, with higher expression in the low-risk group than in the high-risk group. Hence, further studies are needed to explore the relationship between these two genes (IER3 and GOT1) and the prognosis of patients with $\mathrm{CC}$.

Interestingly, we also found some divergences in immune cell infiltration between the high- and low-risk groups, suggesting that glycolysis may be correlated with tumour immunity. In addition, several previous studies 
have elucidated the relationship between glycolysis and immunity. Excess glycolysis will lead to acidic tumor microenvironment, affect the infiltration of immune cells in varying degrees, and contribute to the survival of cancer cells [45]. Cascone et al. found that in patients with melanoma and NSCLC, high glycolytic activity will be accompanied by poor immune cell infiltration, such as cytoxic $\mathrm{T}$ cells, $\mathrm{T}$ helper cells, memory $\mathrm{T}$ cells, macrophases, or natural killer (NK) cells reduction [46]. Li et al. found that high glycolysis group had a higher immunescore and higher TIL percentage in breast cancer, however, the immune cells with anti-tumor effect were not enriched, and concluded that high glycolysis was associated with immunosuppression of tumor microenvironment [47]. In our study, the expression levels of CD8 T cells and resting mast cells were significantly higher in the low-risk group. CD8 T cells, to our knowledge, play a pivotal role in the control of tumour cell growth, and their relationship with the prognosis of patients with $\mathrm{CC}$ has also been confirmed in a previous study $[48,49]$. For mast cells, some studies have found that mast cell infiltration in CC indicates poor clinical prognosis [49-52], which was consistent with our findings that resting mast cells were highly expressed in the low-risk group and that activated mast cells were highly expressed in the high-risk group. These findings are consistent with previous studies and to some extent explain why patients in the low-risk group have better survival outcomes.

It is undeniable that this study does have some defects. On the one hand, we obtained clinical gene information for only 273 cases of CC, and the sample size was not large enough. On the other hand, it would be better to have an external validation cohort to verify the accuracy of the prediction model rather than the internal validation cohort. However, we verified these six DEGRGs between tumor and normal tissues, which to some extent compensated for this defect.

In summary, we first identified the relationship between the GRG signature and the prognosis of patients with $\mathrm{CC}$ using bioinformatics methods and found that patients in the high-risk group had significantly lower OS than those in the low-risk group. Furthermore, we found some relationships between the infiltration of immune cells and glycolysis.

\section{Conclusion}

In brief, we constructed a six-gene signature (HSPA5, ANGPTL4, PFKM, GOT1, IER3 and PFKFB4) to predict the prognosis of patients with $\mathrm{CC}$, which was also a prognostic factor independent of clinicopathological features. These findings will provide us with new insights into the role of glycolysis in $\mathrm{CC}$, guiding individualized treatment for patients with CC.

\section{Supplementary Information}

The online version contains supplementary material available at https://doi. org/10.1186/s12885-020-07598-3.

Additional file 1: Table S1. The mRNA expression profiles.

Additional file 2: Table S2. The clinical information of 273 patients with CC from the UCSC database.

Additional file 3: Table S3. The specifc PCR primers of six mRNAs.

\begin{abstract}
Abbreviations
CC: Cervical cancer; HPV: Human papillomavirus; TLG: Total lesion glycolysis; RFS: Recurrence-free survival; OS: Overall survival; PFS: Progression-free survival; GAPDH: Glyceraldehyde-3-phosphate dehydrogenase; GRG: Glycolysis-related gene; UCSC: University of California Santa Cruz; DEGR Gs: Differentially expressed glycolysis-related genes; GO: Gene Ontology; KEGG: Kyoto Encyclopedia of Genes and Genomes; AJCC: American Joint Committee on Cancer; ROC: Receiver operating characteristic; HIF: Hypoxia inducible factor; GLUT1: Glucose transporter type 1;

PFKM: Phosphofructokinase; EGFRs: Epidermal growth factor receptors; IER3: Immediate early response gene 3; GOT1: Glutamate oxaloacetate transaminase 1; PDAC: Pancreatic ductal adenocarcinoma
\end{abstract}

\section{Acknowledgments}

Not applicable.

\section{Authors' contributions}

XJY conceived and designed the study with LYC and CH. LYC and SSY drafted the manuscript and analyzed the data with $L X L$ and $C H$. XBY and $J H C$ handled the picture and article format. LWF, FL, XL and CZ reviewed the data. All authors have read and approved the final published manuscript.

\section{Funding}

This study was supported by funds from the National Natural Science Foundation of China No. 81503293 (X. J. Y.). The funder had no role in study design, analysis, interpretation of data, the writing of the manuscript and the decision to submit the article for publication.

\section{Availability of data and materials}

The datasets generated and/or analysed during the current study are available in the the University of California Santa Cruz (UCSC) Database and Molecular Signatures Database, [http://xena.ucsc.edu/, https://www.gseamsigdb.org/gsea/msigdb/].

\section{Ethics approval and consent to participate}

Ethical approval for this study was obtained from the Ethics Committee of the First Affiliated Hospital of Wenzhou Medical University, Clinical research ethics review (2019) No.(118). And written informed consent was obtained from all patients.

\section{Consent for publication}

Not applicable.

\section{Competing interests}

The authors declare that they have no competing interests.

\section{Author details}

${ }^{1}$ Department of Obstetrics and Gynecology, The First Affiliated Hospital of Wenzhou Medical University, 2 Fuxue Road, Wenzhou, Zhejiang 325000, P.R. China. ${ }^{2}$ Department of Orthopaedic Surgery, The Affiliated Hospital of Qingdao University, Qingdao 266071, China. ${ }^{3}$ Department of Chemoradiation Oncology, The First Affiliated Hospital of Wenzhou Medical University, Wenzhou, Zhejiang, P.R. China. ${ }^{4}$ Department of Dermatology, The First Affiliated Hospital of Wenzhou Medical University, Wenzhou, Zhejiang, P.R. China. 
Received: 9 May 2020 Accepted: 30 October 2020 Published online: 23 November 2020

\section{References}

1. Bray F, Ferlay J, Soerjomataram I, Siegel RL, Torre LA, Jemal A. Global cancer statistics 2018: GLOBOCAN estimates of incidence and mortality worldwide for 36 cancers in 185 countries. CA Cancer J Clin. 2018;68(6):394-424.

2. Shrestha AD, Neupane D, Vedsted P, Kallestrup P. Cervical Cancer prevalence, incidence and mortality in low and middle income countries: a systematic review. Asian Pac J Cancer Prev. 2018;19(2):319-24.

3. Frenel JSLTC, O'Neil B, Ott PA, Piha-Paul SA, Gomez-Roca C, van Brummelen EMJ, Rugo HS, Thomas S, Saraf S. Safety and efficacy of Pembrolizumab in advanced, programmed death ligand 1-positive cervical Cancer results from the phase Ib KEYNOTE-028 trial. J Clin Oncol. 2017;35(36):4035-61.

4. Pfaendler KS, Tewari KS. Changing paradigms in the systemic treatment of advanced cervical cancer. Am J Obstet Gynecol. 2016;214(1):22-30.

5. Liontos M, Kyriazoglou A, Dimitriadis I, Dimopoulos MA, Bamias A. Systemic therapy in cervical cancer: 30 years in review. Crit Rev Oncol Hematol. 2019; 137:9-17.

6. Vander Heiden MG, Cantley LC, Thompson CB. Understanding the Warburg effect: the metabolic requirements of cell proliferation. Science. 2009; 324(5930):1029-33.

7. Martinez-Ramirez I, Carrillo-Garcia A, Contreras-Paredes A, Ortiz-Sanchez E, Cruz-Gregorio A, Lizano M. Regulation of cellular metabolism by high-risk human papillomaviruses. Int J Mol Sci. 2018;19(7):1839.

8. Leray H, Gabiache E, Courbon F, Brenot-Rossi I, Colineaux H, Lepage B, et al. FDG-PET/CT identifies predictors of survival in patients with locally advanced cervical carcinoma and Para-aortic lymph node involvement to increase treatment. J Nucl Med. 2020;61:1442.

9. Carpenter DJ, Jacobs CD, Wong TZ, Craciunescu O, Chino JP. Changes on midchemoradiation therapy fluorodeoxyglucose positron emission tomography for cervical cancer are associated with prognosis. Int J Radiation Oncol Biol Phys. 2019;105(2):356-66.

10. Leseur J, Roman-Jimenez G, Devillers A, Ospina-Arango JD, Williaume D, Castelli J, et al. Pre- and per-treatment 18F-FDG PET/CT parameters to predict recurrence and survival in cervical cancer. Radiother Oncol. 2016; 120(3):512-8.

11. Liu C, Wang X, Zhang Y. The roles of HK2 on tumorigenesis of cervical Cancer. Technol Cancer Res Treat. 2019;18:1533033819871306.

12. Recondo G, Mezquita L, Bigot L, Galissant J, Frias RL, André F, et al. Preliminary results on mechanisms of resistance to ALK inhibitors: A prospective cohort from the MATCH-R study. Ann Oncol. 2018;29:vi21.

13. Kim JWKS, Han SM, Paik SY, Hur SY, Kim YW, Lee JM, Namkoong SE. Increased glyceraldehyde-3-phosphate dehydrogenase gene expression in human cervical cancers. Gynecol Oncol. 1998;71(2):266-9.

14. Zhang L, Zhang Z, Yu Z. Identification of a novel glycolysis-related gene signature for predicting metastasis and survival in patients with lung adenocarcinoma. J Transl Med. 2019;17(1):423.

15. Jiang LZL, Bi J, Guan Q, Qi A, Wei Q, He M, Wei M, Zhao L. Glycolysis gene expression profilings screen for prognostic risk signature of hepatocellular carcinoma. Aging (Albany NY). 2019;11(23):10861-82.

16. Tian G, Li G, Liu P, Wang Z, Li N. Glycolysis-based genes associated with the clinical outcome of pancreatic ductal adenocarcinoma identified by the Cancer genome atlas data analysis. DNA Cell Biol. 2020;39(3):417-27.

17. Wang $Z \mathrm{H}$, Zhang $Y Z$, Wang $Y S$, Ma XX. Identification of novel cell glycolysis related gene signature predicting survival in patients with endometrial cancer. Cancer Cell Int. 2019;19:296.

18. Newman AM, Liu CL, Green MR, Gentles AJ, Feng W, Xu Y, Hoang CD, Diehn M, Alizadeh AA. Robust enumeration of cell subsets from tissue expression profiles. Nat Methods. 2015;12(5):453-7.

19. Krzywinski M, Schein J, Birol I, Connors J, Gascoyne R, Horsman D, Jones SJ, Marra MA. Circos: an information aesthetic for comparative genomics. Genome Res. 2009;19(9):1639-45.

20. Maxwell PHDG, Gleadle JM, Nicholls LG, Harris AL, Stratford IJ, Hankinson O, Pugh CW, Ratcliffe PJ. Hypoxia-inducible factor-1 modulates gene expression in solid tumors and influences both angiogenesis and tumor growth. Proc Natl Acad Sci U S A. 1997;94(15):8104-9.

21. Parks SK, Chiche J, Pouyssegur J. Disrupting proton dynamics and energy metabolism for cancer therapy. Nat Rev Cancer. 2013;13(9):611-23.
22. Ding QDS, Wang R, Zhang K, Wang H, Zhou X, Wang J, Wong K, Long $Y$, Zhu S. A nine-gene signature related to tumor microenvironment predicts overall survival with ovarian cancer. Aging (Albany NY). 2020;12(6):4879-95.

23. Hu D, Jiang L, Luo S, Zhao X, Hu H, Zhao G, Tang W. Development of an autophagy-related gene expression signature for prognosis prediction in prostate cancer patients. J Transl Med. 2020;18(1):160.

24. AS L. The glucose-regulated proteins: stress induction and clinical applications. Trends Biochem Sci. 2001;26(8):504-10.

25. Jamora CDG, Lee AS. Inhibition of tumor progression by suppression of stress protein GRP78/BiP induction in fibrosarcoma B/C10ME. Proc Natl Acad Sci U S A. 1996;93(15):7690-4.

26. Li H, Ge C, Zhao F, Yan M, Hu C, Jia D, et al. Hypoxia-inducible factor 1 alpha-activated angiopoietin-like protein 4 contributes to tumor metastasis via vascular cell adhesion molecule-1/integrin beta1 signaling in human hepatocellular carcinoma. Hepatology. 2011;54(3):910-9.

27. Padua D, Zhang XH, Wang Q, Nadal C, Gerald WL, Gomis RR, Massague J. TGFbeta primes breast tumors for lung metastasis seeding through angiopoietin-like 4. Cell. 2008;133(1):66-77.

28. Chiang KH, Shieh JM, Shen CJ, Chang TW, Wu PT, Hsu JY, Tsai JP, Chang WC, Chen BK. Epidermal growth factor-induced COX-2 regulates metastasis of head and neck squamous cell carcinoma through upregulation of angiopoietin-like 4. Cancer Sci. 2020;111(6):2004-15.

29. Yang W, Khoury E, Guo Q, Prabhu SA, Emond A, Huang F, et al. MNK1 signaling induces an ANGPTL4-mediated gene signature to drive melanoma progression. Oncogene. 2020;39(18):3650-65.

30. Rahmani F, Hasanzadeh M, Hassanian SM, Khazaei M, Esmaily H, Asef-Agah SA, Naghipour A, AF G, Avan A. Association of a genetic variant in the angiopoietin-like protein 4 gene with cervical cancer. Pathol Res Pract. 2020; 216(7):153011.

31. Sun CM, Xiong DB, Yan Y, Geng J, Liu M, Yao XD. Genetic alteration in phosphofructokinase family promotes growth of muscle-invasive bladder cancer. Int J Biol Markers. 2016;31(3):e286-93.

32. Ahsan H, Halpern J, Kibriya MG, Pierce BL, Tong L, Gamazon E, et al. A genome-wide association study of early-onset breast cancer identifies PFKM as a novel breast cancer gene and supports a common genetic spectrum for breast cancer at any age. Cancer Epidemiol Biomark Prev. 2014;23(4):658-69.

33. Mattarocci S, Abbruzzese C, Mileo AM, Carosi M, Pescarmona E, Vico C, et al. Identification of pivotal cellular factors involved in HPV-induced dysplastic and neoplastic cervical pathologies. J Cell Physiol. 2014;229(4):463-70.

34. Goncalves MD, Cantley LC. A glycolysis outsider steps into the Cancer spotlight. Cell Metab. 2018;28(1):3-4.

35. Dasgupta S, Rajapakshe K, Zhu B, Nikolai BC, Yi P, Putluri N, et al. Metabolic enzyme PFKFB4 activates transcriptional coactivator SRC-3 to drive breast cancer. Nature. 2018;556(7700):249-54.

36. Ros S, Santos CR, Moco S, Baenke F, Kelly G, Howell M, Zamboni N, Schulze A. Functional metabolic screen identifies 6-phosphofructo-2-kinase/fructose2,6-biphosphatase 4 as an important regulator of prostate cancer cell survival. Cancer Discov. 2012;2(4):328-43.

37. Goidts V, Bageritz J, Puccio L, Nakata S, Zapatka M, Barbus S, et al. RNAi screening in glioma stem-like cells identifies PFKFB4 as a key molecule important for cancer cell survival. Oncogene. 2012;31(27):3235-43.

38. Arlt A, Schafer $\mathrm{H}$. Role of the immediate early response 3 (IER3) gene in cellular stress response, inflammation and tumorigenesis. Eur J Cell Biol. 2011;90(6-7):545-52.

39. HM S. Gomori-positive astrocytes: biological properties and implications for neurologic and neuroendocrine disorders. Glia. 1991;4(4):365-77.

40. Jin H, Lee K, Kim YH, Oh HK, Maeng YI, Kim TH, Suh DS, Bae J. Scaffold protein FHL2 facilitates MDM2-mediated degradation of IER3 to regulate proliferation of cervical cancer cells. Oncogene. 2016;35(39):5106-18.

41. Jin H, Suh DS, Kim TH, Yeom JH, Lee K, Bae J. IER3 is a crucial mediator of TAp73beta-induced apoptosis in cervical cancer and confers etoposide sensitivity. Sci Rep. 2015;5:8367.

42. Abrego J, Gunda V, Vernucci E, Shukla SK, King RJ, Dasgupta A, et al. GOT1mediated anaplerotic glutamine metabolism regulates chronic acidosis stress in pancreatic cancer cells. Cancer Lett. 2017:400:37-46.

43. Birsoy K, Wang T, Chen WW, Freinkman E, Abu-Remaileh M, Sabatini DM. An essential role of the mitochondrial Electron transport chain in cell proliferation is to enable aspartate synthesis. Cell. 2015;162(3):540-51.

44. Melendez-Rodriguez F, Urrutia AA, Lorendeau D, Rinaldi G, Roche O, Bogurcu-Seidel N, et al. HIF1alpha suppresses tumor cell proliferation 
through inhibition of aspartate biosynthesis. Cell Rep. 2019;26(9):2257-65

e2254.

45. Gill KS, Fernandes P, O'Donovan TR, McKenna SL, Doddakula KK, Power DG, Soden DM, Forde PF. Glycolysis inhibition as a cancer treatment and its role in an anti-tumour immune response. Biochim Biophys Acta. 2016;1866(1): 87-105.

46. Cascone T, McKenzie JA, Mbofung RM, Punt S, Wang Z, Xu C, et al. Increased tumor glycolysis characterizes immune resistance to adoptive $T$ cell therapy. Cell Metab. 2018;27(5):977-87 e974.

47. Li W, Xu M, Li Y, Huang Z, Zhou J, Zhao Q, et al. Comprehensive analysis of the association between tumor glycolysis and immune/inflammation function in breast cancer. J Transl Med. 2020;18(1):92.

48. de Vos van Steenwijk PJ, Ramwadhdoebe TH, Goedemans R, Doorduijn EM, van Ham JJ, Gorter A, et al. Tumor-infiltrating CD14-positive myeloid cells and CD8-positive T-cells prolong survival in patients with cervical carcinoma. Int J Cancer. 2013;133(12):2884-94.

49. Yang $S$, Wu Y, Deng Y, Zhou L, Yang P, Zheng Y, et al. Identification of a prognostic immune signature for cervical cancer to predict survival and response to immune checkpoint inhibitors. Oncoimmunology. 2019;8(12): e1659094.

50. Jiang LHY, Shen Q, Ding S, Jiang W, Zhang W, Zhu X. Role of mast cells in gynecological neoplasms. Front Biosci (Landmark Ed). 2013;18:773-81.

51. Ferrandina GRF, Legge F, Gessi M, Salutari V, Distefano MG, Lauriola L, Zannoni GF, Martinelli E, Scambia G. Prognostic role of the ratio between cyclooxygenase-2 in tumor and stroma compartments in cervical cancer. Clin Cancer Res. 2004;10(9):3117-23.

52. Ferrandina G, Lauriola L, Zannoni GF, Distefano MG, Legge F, Salutari V, et al. Expression of cyclooxygenase-2 (COX-2) in tumour and stroma compartments in cervical cancer: clinical implications. Br J Cancer. 2002; 87(10):1145-52.

\section{Publisher's Note}

Springer Nature remains neutral with regard to jurisdictional claims in published maps and institutional affiliations.

Ready to submit your research? Choose BMC and benefit from:

- fast, convenient online submission

- thorough peer review by experienced researchers in your field

- rapid publication on acceptance

- support for research data, including large and complex data types

- gold Open Access which fosters wider collaboration and increased citations

- maximum visibility for your research: over $100 \mathrm{M}$ website views per year

At $\mathrm{BMC}$, research is always in progress.

Learn more biomedcentral.com/submissions 